\title{
Optimal Scenario Reduction Framework based on Distance of Uncertainty Distribution and Output Performance: II. Sequential Reduction
}

\author{
Zukui $\mathrm{Li}^{1}$ and Christodoulos A. Floudas ${ }^{* 2}$ \\ ${ }^{1}$ Departmet of Chemical and Materials Engineering, University of Alberta, Edmonton, \\ AB, T6G2V4, Canada \\ ${ }^{2}$ Texas A\&M Energy Institute, Texas A\&M University, College Station, TX 77843, USA
}

\begin{abstract}
In this paper, a novel sequential scenario reduction framework for general optimization problem is proposed. The proposed method extends the previous work (Li and Floudas, 2014) and aims to tackle optimization problems with a large number of uncertain parameters and a huge number of scenarios generated from the factorial combination. The proposed method first ranks the uncertain parameters based on their effects on the optimal objective using global sensitivity analysis. Then, the parameters are sequentially considered in generating uncertainty scenarios. This method can essentially reduce the computational efforts needed for evaluating the objective values of all scenarios, which is often impractical for a huge number of scenarios. Criteria for quantifying the quality of scenario reduction are also proposed based on robust optimization and scenario optimization. Case studies are presented to illustrate the sequential scenario reduction framework and the results verify the efficiency of the proposed approach.
\end{abstract}

Keywords: optimal scenario reduction, sequential framework, uncertainty, mixed integer linear optimization

\footnotetext{
* Author to whom correspondence should be addressed.

Email: floudas@tamu.edu; Tel: 979-458-0253
} 


\section{Introduction}

In many applications of optimization under uncertainty, uncertainty is represented using scenarios. Given continuous or discrete distributions of uncertain parameters, scenarios can be generated to represent the distribution of random variables. Scenario generation has been widely studied in the past and various methods have been proposed (Pflug, 2001; Hochreiter and Pflug, 2007; Mirkov and Pflug, 2007, Calfa et al., 2014), such as sampling based method, statistic moment matching method, and stochastic process simulation method. In general, it requires a large number of scenarios to accurately represent the uncertainty. However, for many optimization applications, including a large number of scenarios into the model will make the problem intractable. Hence, scenario reduction has received a lot of attention in the past. Most existing scenario reduction approaches have focused on the two-stage or multistage stochastic programming problem. Specifically, scenario reduction methods have been discussed in (Dupacova et al., 2003; Heitsch and Romisch, 2003; Heitsch and Romisch, 2007; Henrion et al., 2008) for two stage stochastic programming problems and in (Heitsch et al., 2006; Heitsch and Romisch, 2009; Pennanen, 2009; Pflug, 2009) for multistage stochastic programming problems.

Applications of scenario reduction are still in general restricted and we present a few literature approaches. Sumaili et al. (2011) proposed a technique able to represent the wind power forecasting uncertainty by a set of representative scenarios capable of characterizing the probability density function of the wind power forecast, which will allow the reduction of the computational burden in stochastic models that require scenario reduction. Xue et al. (2011) studied an approach for extracting representative spatio-temporal weather-impact scenarios and the corresponding probabilities of occurrence, from a large stochasticallygenerated ensemble of possible scenarios. Costa et al. (2006) studied the stochastic hydrothermal scheduling optimization problem, and used principal component analysis to reduce the effective dimensionality of the scenario specification problem so that a discretization technique can be used in a smaller dimensional space. Karuppiah et al. (2010) presented a heuristic strategy of selecting scenarios based on a simple criterion that the sum

of the probabilities of the new scenarios in which the uncertain parameter value appears is equal to its original probability value and applied it to chemical process planning problems. 
De oliveira et al. (2010) applied principal component analysis to reduce the effective dimensionality of the scenario specification problem to address the mid-term operation planning of hydro-thermal power systems.

While traditional methods focus more on the distribution of the uncertain parameters, the proposed work in the first part of the series of paper (Li and Floudas, 2014) incorporates the performance of the output space of decision making. To achieve this objective, not only the probabilistic distance between the original and the reduced distribution for the input space were considered, but also the best, worst and expected performance on the output performance were included. The optimal scenario selection/reduction problem was based on minimizing the Kantorovich distance between the probability distribution represented by the original scenario set and the probability distribution represented by the selected subset of scenarios, and the difference between the expected performance, best performance, and the worst performance.

One major limitation of the existing scenario reduction algorithms is that they cannot address a huge number of scenarios. In reality, however, uncertainty in even a moderate number of parameters can lead to a huge number of scenarios. For example, 30 parameters with each taking 10 discrete values will lead to $10^{30}$ scenarios with a factorial combination, which is beyond the capability of most existing algorithms or tools. As far as the authors know, so far there is not yet a readily available tool for scenario reduction from such a big number of scenarios.

The scenario reduction algorithm introduced in ( $\mathrm{Li}$ and Floudas, 2014) demonstrates superior performance in both the distance between the uncertainty distribution and the performance of the optimization solution's distribution. However, that method is limited by the size of the problem so that it can only be applied to solve problems with a relatively small number of scenarios (i.e., up to 5000 scenarios). Although a decomposition scheme can be applied to address relatively larger cases, the issue of "dimensional explosion" still exists. In this paper, to make a realistic scenario reduction tool capable of addressing a huge number of scenarios generated from a large number of uncertain parameters, we propose a 
novel sequential scenario reduction framework. To check the quality of the scenario reduction, we also propose several novel criteria to quantify the gap between the performance of the original scenarios and that of the reduced scenarios.

The rest of the paper is organized as follows. In Section 2, we analyze the problem size and introduce optimization based criteria to quantify the quality of scenario reduction. In Section 3, we present the sequential reduction framework through an illustration example. In Section 4, we investigate the method using case studies and Section 5 concludes the paper.

\section{Problem description}

Consider the following general optimization problem

$$
\begin{array}{ll}
\max _{x} & f(x, \theta) \\
\text { s.t. } & g(x, \theta) \leq 0
\end{array}
$$

where the parameters $\theta=\left[\theta_{1}, \cdots, \theta_{k}\right] \in \mathbf{R}^{k}$ are subject to uncertainty. It is assumed that each uncertain parameter $\theta_{1}, \ldots, \theta_{k}$ follows independent discrete distribution, and the joint uncertainty distribution is represented by a set of scenarios $U=\left\{\theta^{1}, \theta^{2}, \ldots, \theta^{n}\right\}$, where $\theta^{i}=\left[\theta_{1}^{i}, \cdots, \theta_{k}^{i}\right] i=1, \ldots, n$. For each scenario, it is assumed that the optimal objective of the optimization problem can be obtained by solving the following deterministic problem

$$
\begin{array}{rl}
f_{i}=\max _{x} & f\left(x, \theta^{i}\right) \\
& \text { s.t. } g\left(x, \theta^{i}\right) \leq 0
\end{array}
$$

Notice that slack variables can be incorporated into the constraints and penalty terms of the slack variables can be added to the objective function of the original optimization problem to ensure the feasibility of problem (2) under every scenario.

In this work, the problem under investigation is to find a subset of the realizations $S$ İ $U$, such that the distribution of the optimal solution from all the original scenarios and the selected subset of scenarios are as similar as possible. 


\subsection{Reduction of scenarios generated from factorial combination}

Assume that each uncertain parameter $\theta_{1}, \ldots, \theta_{k}$ can take a fixed number of discrete levels. The uncertainty space is represented by a factorial combination of different levels of all the uncertain parameters. For typical problems listed in Table 1, the number of scenarios quickly increases to a huge number as the number of parameters and the number of discretization levels increase.

Table 1. Typical number of scenarios from factorial combination

\begin{tabular}{|c|c|c|c|c|}
\hline \multirow{2}{*}{} & \multicolumn{4}{|c|}{ Discretization level } \\
\cline { 2 - 5 } & 3 & 5 & 7 & 9 \\
\hline 5 parameters & 243 & 3125 & 16807 & 59049 \\
\hline 10 parameters & $5.9 \times 10^{4}$ & $9.8 \times 10^{6}$ & $2.8 \times 10^{8}$ & $3.5 \times 10^{9}$ \\
\hline 20 parameters & $3.5 \times 10^{9}$ & $9.5 \times 10^{13}$ & $8.0 \times 10^{16}$ & $1.2 \times 10^{19}$ \\
\hline 50 parameters & $7.2 \times 10^{23}$ & $8.9 \times 10^{34}$ & $1.8 \times 10^{42}$ & $5.2 \times 10^{47}$ \\
\hline 100 parameters & $5.2 \times 10^{47}$ & $7.9 \times 10^{69}$ & $3.2 \times 10^{84}$ & $2.7 \times 10^{95}$ \\
\hline
\end{tabular}

The scenario reduction method introduced in Li and Floudas (2014) can be simply represented as Figure 1. It aims to find the desired number of scenarios from superset via the solution of one mixed integer linear optimization model. However, it is impractical to address the typical problems listed in Table 1 using the single stage scenario reduction algorithm. First, the single stage scenario reduction algorithm can only solve problems with up to 5000 scenarios because of the combinatorial complexity of the mixed integer optimization model. Second, it is impractical to evaluate the optimal objective values and pairwise dissimilarities between scenarios for a huge number of scenarios.

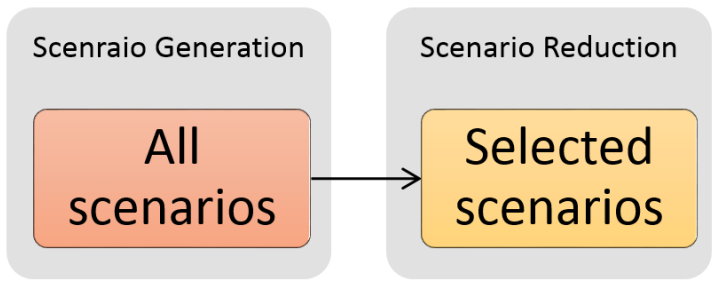

Figure 1. Single stage scenario reduction scheme 
One possible strategy to address the large size of the scenario set is to apply a decomposition based reduction scheme as shown in Figure 2. In this type of approach, all the scenarios are generated in the first step. Then the superset of scenarios is decomposed and grouped into subsets (e.g., randomly) with an appropriate size that can be handled by single scenario reduction. For each subset, the single scenario reduction method of (Li and Floudas, 2014) can be applied. Their reduction results are finally aggregated to generate the final selection.

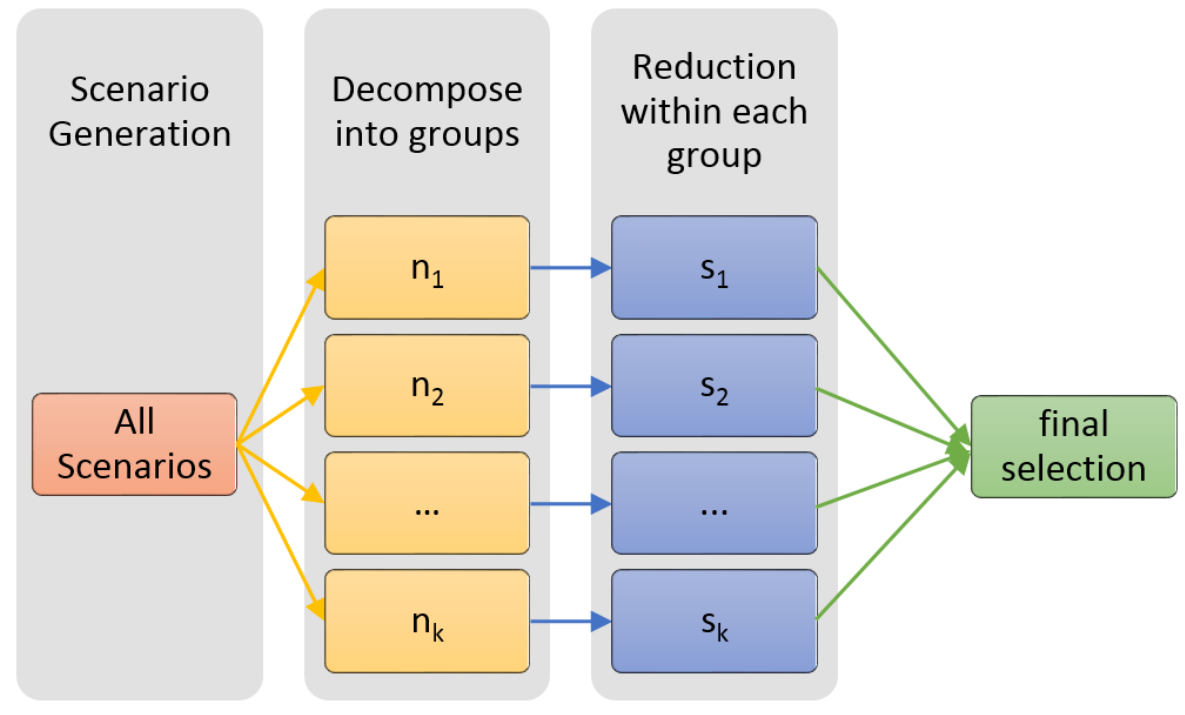

Figure 2. Decomposition based reduction

However, even using the above decomposition strategy, the issue of solution complexity still exists, and the reduction of a huge number of scenarios is still challenging because the number of scenarios increases exponentially with the number of parameters. For example, to handle $9.5 \times 10^{13}$ scenarios (generated from 20 parameters, each with 5 levels) with subset size 5000 , the total number of subsets is $1.9 \times 10^{10}$. That is, $1.9 \times 10^{10}$ single stage scenario reduction problems need to be solved first. Furthermore, even we just select 100 scenarios from each subset (5000 scenarios), the aggregated results will lead to $1.9 \times 10^{12}$ scenarios, which is still a large number and further decomposition and reduction are needed.

To address the above issue, we propose a sequential reduction framework, as detailed in the following sections. The proposed method will not suffer from the dimensionality issue explained above. The method can significantly reduce the computational efforts needed for 
evaluating a huge number of scenarios and provides a practical way to perform scenario reduction for realistic large scale problems.

\subsection{Quantify the quality of scenario reduction}

In the first part of the series of paper (Li and Floudas, 2014), to check the quality of the single stage scenario reduction, the objective values of all the scenarios were solved and the original distribution has been generated for validation. However, in this paper, because of the huge number of scenarios, it is impractical to generate the original distribution from all scenarios for comparison. To quantify the quality of the sequential scenario reduction, an alternative method is proposed in this work based on the following properties for scenario optimization and robust optimization.

Property 1. Consider a set $S$ which contains $s$ scenarios: $S=\left\{\theta^{1}, \theta^{2}, \ldots, \theta^{s}\right\}$, where $\theta^{L} \leq \theta^{i} \leq \theta^{U}, i=1, \cdots, s$, the best performance of the optimization problem among all the scenarios is given by

$$
f_{B P}^{S}=\max _{\theta \in\left\{\theta^{1}, \theta^{2}, \ldots, \theta^{s}\right\}}\left\{\begin{array}{l}
\max _{x} f(x, \theta) \\
\text { s.t. } g(x, \theta) \leq 0
\end{array}\right\}
$$

An upper bound of the above problem's optimal solution is given by the solution of the following relaxed optimization problem where $\theta$ are considered as variables:

$$
\begin{aligned}
\hat{f}_{B P}=\max _{x, \theta} & f(x, \theta) \\
\text { s.t. } & g(x, \theta) \leq 0 \\
& \theta^{L} \leq \theta \leq \theta^{U}
\end{aligned}
$$

Proof: Notice that the feasible solution space of problem (3) is a subset of problem (4). Furthermore, the two problems have the same maximization objective. So the optimal solution of (4) is an upper bound of that of problem (3).

Property 2. Consider a superset of scenarios $U$ and a subset of selected scenarios $S \subset U$, the following relationship holds

$$
f_{B P}^{S} \leq f_{B P}^{U} \leq \hat{f}_{B P}
$$


where $f_{B P}^{S}$ is the optimal solution of best performance optimization problem (3) under selected scenarios, $f_{S O}^{U}$ is the optimal solution of the best performance optimization problem (3) under all scenarios.

Proof: First, Property 1 shows that $f_{B P}^{S} \leq \hat{f}_{B P}, f_{B P}^{U} \leq \hat{f}_{B P}$. Second, since $S \subset U$, the feasible space of problem (3) with set $S$ is smaller than the feasible space of problem (3) with set $U$, hence $f_{B P}^{S} \leq f_{B P}^{U}$.

Property 3. Given $s$ scenarios $\theta^{1}, \theta^{2}, \ldots, \theta^{s}$, where $\theta^{L} \leq \theta^{i} \leq \theta^{U}, i=1, \cdots, s$, consider the following scenario optimization problem

$$
\begin{aligned}
f_{S O}^{S}= & \max _{x} f(x) \\
\text { s.t. } & g\left(x, \theta^{i}\right) \leq 0 \quad \forall i=1, \cdots, S
\end{aligned}
$$

A lower bound of the optimal solution of (6) is given by the solution of the following robust optimization problem

$$
\begin{aligned}
f_{R O}= & \max _{x} f(x) \\
& \text { s.t. }\left\{\max _{\theta \in\left[\theta^{L}, \theta^{U}\right]} g(x, \theta)\right\} \leq 0
\end{aligned}
$$

Proof: The feasible space of problem (7) is smaller than that of the problem (6), because (7) requires that the constraints be satisfied for all the possible scenarios within the uncertain parameter space, while problem (6) enforces that the constraints should be satisfied for a limited number of scenarios within the uncertainty parameter space. Furthermore, since (6) and (7) have the same objective for maximization, optimal solution of (7) provides a lower bound for (6).

Property 4. Consider a superset of scenarios $U$ and a subset of selected scenarios $S \subset U$, we have the following relationship

$$
f_{R O} \leq f_{S O}^{U} \leq f_{S O}^{S}
$$

where $f_{S O}^{S}$ is the optimal solution of the scenario optimization problem (6) under selected scenarios, $f_{S O}^{U}$ is the optimal solution of the scenario optimization problem (6) under all the original scenarios. 
Proof: First, Property 3 shows that $f_{R O} \leq f_{S O}^{S}, f_{R O} \leq f_{S O}^{U}$. Second, since $S \subset U$, the feasible space of problem (7) with set $S$ is larger than the feasible space of problem (7) with set $U$, hence $f_{S O}^{U} \leq f_{S O}^{S}$.

Based on the above property (5), the following relationship can be derived

$$
0 \leq f_{B P}^{U}-f_{B P}^{S} \leq \hat{f}_{B P}-f_{B P}^{S}
$$

Similarly, property (8) leads to the following inequality

$$
f_{R O}-f_{S O}^{S} \leq f_{S O}^{U}-f_{S O}^{S} \leq 0
$$

In this paper, the following gaps are defined to quantify the quality of scenario reduction

$$
\begin{aligned}
& e r r_{1}=\hat{f}_{B P}-f_{B P}^{S} \\
& e r r_{2}=f_{R O}-f_{S O}^{S}
\end{aligned}
$$

To make the distribution of the optimal solution from all the original scenarios $U$ and the selected subset of scenarios $S$ as similar as possible, the two differences $f_{B P}^{U}-f_{B P}^{S}$ and $f_{S O}^{U}-f_{S O}^{S}$ should be as close to zero as possible. Since it is impractical to evaluate $f_{B P}^{U}$ and $f_{S O}^{U}$ because of the huge number of scenarios, we use the gaps $e r r_{1}$ and $e r r_{2}$ to quantify the difference $f_{B P}^{U}-f_{B P}^{S}$ and $f_{S O}^{U}-f_{S O}^{S}$ : a small gap $e r r_{1}=\hat{f}_{B P}-f_{B P}^{S}$ means a small difference $f_{B P}^{U}-f_{B P}^{S}$ and similarly a small gap $e r r_{2}=f_{R O}-f_{S O}^{S}$ ensures that the difference $f_{S O}^{U}-f_{S O}^{S}$ is close to zero. Thus, the quality of scenario reduction can be validated from the value err and $\mathrm{err}_{2}$.

\section{Sequential scenario reduction framework}

The proposed sequential reduction algorithm is based on the optimal single stage scenario reduction algorithm presented in the first part of the series of paper (Li and Floudas, 2014). It applies the single stage scenario reduction sequentially to reduce the number of scenarios. The uncertain parameters are not considered one-at-a-time. Instead, they are investigated 
sequentially based on their importance on affecting the optimal value of the optimization problems. The importance of the parameters can be obtained by ranking the global sensitivity of each parameter.

\subsection{Parameters ranking}

Global sensitivity analysis can be performed to identify the importance ranking of the uncertain parameters. The sensitivity of an input illustrates the effect that a variation on it will have on the output. Input of higher sensitivity will result in a greater variation of the output. The Morris method and variance based methods are typical global sensitivity analysis methods.

\section{Morris Method}

The Morris method identifies the influence of input variables on a model's output with a limited number of model simulations. It is based on the "one-factor-at-a-time" assumption that if all variables are changed by the same percentage, the variable that exhibits the largest variation in the output is the most sensitive (Morris, 1991).

In this method, a multiple number of trajectories through the parameter space are generated to explore the model output. If the number of parameters is $k$, then a trajectory is a sequence

of $k+1$ points in the parameter space and it satisfies the following rules: (1) starting from a random point in the discretization grid; (2) any two consecutive points differ only for one component.

Each trajectory provides a single estimation of the elementary effect $\left(e e_{i}\right)$ for each model input $\left(q_{i}\right)$. The elementary effect is defined as the changes in output due to changes in a particular input.

$$
e e_{i}=\frac{f\left(\theta_{1}, \theta_{2}, \ldots, \theta_{i-1}, \theta_{i}+\Delta, \theta_{i+1}, \ldots, \theta_{k}\right)-f\left(\theta_{1}, \theta_{2}, \ldots, \theta_{i-1}, \theta_{i}, \theta_{i+1}, \ldots, \theta_{k}\right)}{\Delta}
$$

Along each trajectory, $k$ elementary effects can be computed. The Morris method ensures that the trajectory is designed such that one elementary effect per factor can be computed. 
Two measures are provided by the Morris method based on the elementary effects' distribution: (1) the sensitivity index $\mu$ (mean of the elementary effects) assesses the overall influence of a variable on the model output, including the higher-order and interaction effects. When $\mu$ is high, the variable is highly sensitive as a unit change causes a large deviation of output and vice versa; (2) the standard deviation $\sigma$ (standard deviation of elementary effects) provides a measure that indicates possible interaction of a variable with other variables and/or the variable that has a non-linear effect on the output. In this paper, $\mu$ is used as an index to rank the importance of the parameters.

\section{Variance Based Method}

Variance-based sensitivity analysis methods aim to quantify the amount of variance that each parameter contributes to the unconditional variance of the model output. These methods can quantify interactions between variables. Two types of sensitivity indices can be provided by variance based methods. The first type index is the first order sensitivity index $\mathrm{S}_{\mathrm{i}}$, which estimates of the ratio of the variance due to the $i$-th variable to the variance due to all variables. The second sensitivity measure that can be computed using variance based methods is the total sensitivity index $\mathrm{S}_{\mathrm{Ti}}$, which is defined as the sum of all effects involving the $i$-th variable. Variance based methods include Sobol method (Sobol, 1993), FAST method (Cukier et al., 1973) and its extended version EFAST (Saltelli et al, 1999), etc. Variance based methods are more computationally expensive, since they require larger number of model simulations to estimate multi-dimensional integral. For problems with a large number of parameters, the Morris method has lower computationally cost. Hence, the Morris method is applied in this work.

\subsection{Sequential scenario reduction method}

Based on the ranking of the parameters, the single stage scenario reduction approach introduced in (Li and Floudas, 2014) can be sequentially applied. The complete sequential algorithm can be described as follows. 
Step 1. Rank the uncertain parameters: Based on the contribution of the uncertain parameter to the optimal objective of the optimization problem, rank the parameters using global sensitivity analysis (with a descending importance).

Step 2. Set the reduction parameters: In this step, the sequential scheme is designed to ensure that each single scenario reduction optimization can be efficiently solved. The parameters to be set include the starting number of parameters $N$, the number of scenarios selected in each step $m$, and the terminating point before the last reduction step.

Step 3. Sequential reduction.

a. Generate scenarios by using the parameters under investigation and fixing the rest parameters at nominal values. Calculate the initial probability of each individual scenario.

b. Perform single stage scenario reduction using the approach of Li and Floudas (2014).

c. Save the selected scenarios and their corresponding probabilities.

d. Incorporate the next parameter for scenario reduction, go to step (3a). This step is repeated until all the uncertain parameters have been investigated.

Step 4. Report the final selected scenarios and the corresponding probabilities.

\subsection{Illustration example}

To illustrate the proposed method for sequential scenario reduction, a refinery production optimization problem is considered. A simplified flowchart of the refinery is shown in Figure 3. The refinery optimization problem is formulated as a linear optimization problem as follows (Kendrick et al., 1981):

$$
\begin{aligned}
& \max \sum_{c f} p f_{c f} x_{c f}-\left(\sum_{c r} p r_{c r} u_{c r}+\sum_{c d} \sum_{c r} p d_{c d} u i_{c r, c d}\right)-\sum_{p} \sum_{r \in R} o p_{p} z_{c r, p} \\
& \text { s.t. } \quad \sum_{p \in P} a_{c r, ' c r u d e^{\prime}, p} z_{c r, p}+u_{c r} \geq 0 \quad \forall c r
\end{aligned}
$$




$$
\begin{aligned}
& \sum_{p} a_{c r, c i, p} z_{c r, p}+u i_{c r, c d} \geq \sum_{c f,(c f, c i) \in B P} w_{c r, c i, c f} \quad \forall c r, c i \\
& q s_{c f, q}^{l b} x_{c f} \leq \sum_{c r} \sum_{c i} a t c_{c r, c i, q} w_{c r, c i, c f} \leq q s_{c f, q}^{u b} x_{c f} \quad \forall c f, q \\
& \sum_{p} b_{m, p} \sum_{c r} z_{c r, p} \leq k_{m} \quad \forall m \\
& u_{c r} \leq u r_{c r} \forall c r \\
& x_{c f}=\sum_{c r, c i,(c f, c i) \in B P} w_{c r, c i, c f} \forall c f
\end{aligned}
$$

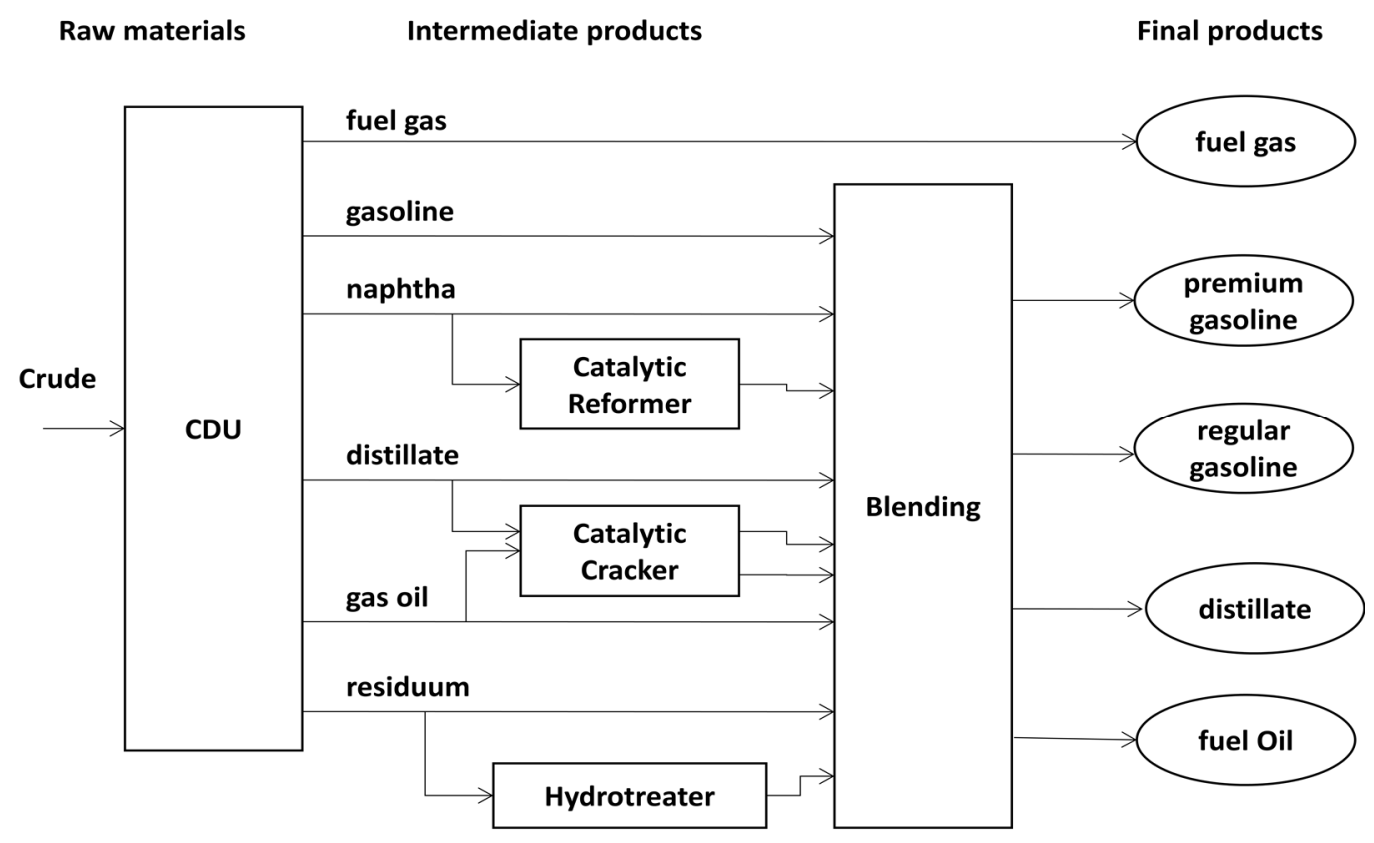

Figure 3. Schematic of product flows in a refinery

A detailed nomenclature for the above problem is given in the Appendix. The objective function (14a) aims to maximize the profit calculated by revenues from the sale of final products, cost of raw materials and operating cost. Constraints (14b) enforce the material balance constraints for intermediates. Constraints (14c) enforce crude oil inputs of crude oil type into all processes are less than the purchases of materials. Constraints (14d) represent quality requirements for final products. Constraints (14e) are capacity constraints for each production unit. Constraints (14f) represent limits (upper bounds) on crude oil purchases and constraints $(14 \mathrm{~g})$ state that final sales must equal the output of the blending process. 
In this example, we consider the uncertainty in the 8 price parameters listed in Table 2, each of them take 5 possible discrete values with different probabilities. Total number of scenarios is $5^{8}=390625$ scenarios. The proposed sequential scenario reduction method is applied to reduce those scenarios.

Table 2. Uncertainty in price data of raw materials and final products

\begin{tabular}{|c|c|c|}
\hline Parameter & Uncertainty level & Probability \\
\hline$p d$ ('butane') & {$\left[\begin{array}{llllll}6.5 & 6.625 & 6.75 & 6.875 & 7.0\end{array}\right]$} & {$\left[\begin{array}{llllll}0.1 & 0.2 & 0.4 & 0.2 & 0.1\end{array}\right]$} \\
\hline $\operatorname{pr}($ 'mid-c') & {$\left[\begin{array}{lllll}7.0 & 7.25 & 7.5 & 7.75 & 8.0\end{array}\right]$} & {$\left[\begin{array}{llllll}0.1 & 0.1 & 0.6 & 0.1 & 0.1\end{array}\right]$} \\
\hline $\operatorname{pr}\left({ }^{\prime} \mathrm{w}-\mathrm{tex}\right.$ ') & {$\left[\begin{array}{lllll}6.0 & 6.25 & 6.5 & 6.75 & 7.0\end{array}\right]$} & {$\left[\begin{array}{lllll}0.1 & 0.1 & 0.5 & 0.15 & 0.15\end{array}\right]$} \\
\hline$p f($ 'premium') & {$\left[\begin{array}{llllll}10 & 10.25 & 10.5 & 10.75 & 11\end{array}\right]$} & {$\left[\begin{array}{lllll}0.1 & 0.1 & 0.6 & 0.1 & 0.1\end{array}\right]$} \\
\hline$p f($ 'regular') & {$\left[\begin{array}{lllll}8.9 & 9.0 & 9.1 & 9.2 & 9.3\end{array}\right]$} & {$\left[\begin{array}{lllll}0.1 & 0.1 & 0.6 & 0.1 & 0.1\end{array}\right]$} \\
\hline$p f$ ('distillate') & {$\left[\begin{array}{lllll}7.5 & 7.6 & 7.7 & 7.8 & 7.9\end{array}\right]$} & {$\left[\begin{array}{llllll}0.05 & 0.05 & 0.8 & 0.05 & 0.05\end{array}\right]$} \\
\hline$p f($ 'fuel-oil') & {$\left[\begin{array}{llllll}6.45 & 6.55 & 6.65 & 6.75 & 6.85\end{array}\right]$} & {$\left[\begin{array}{llllll}0.05 & 0.2 & 0.5 & 0.2 & 0.05\end{array}\right]$} \\
\hline$p f$ ('fuel-gas') & {$\left[\begin{array}{llllll}1.3 & 1.4 & 1.5 & 1.6 & 1.7\end{array}\right]$} & {$\left[\begin{array}{lllll}0.1 & 0.2 & 0.4 & 0.2 & 0.1\end{array}\right]$} \\
\hline
\end{tabular}

In the beginning of the scenario reduction, the parameters' importance are ranked from high to low using the global sensitivity analysis method. The Morris method is applied and the ranking results are shown in Table 3 . Notice that the parameters have also been labeled as P1 to P8 based on the ranking.

Table 3. Ranking results using the Morris method

\begin{tabular}{|c|c|c|c|c|c|c|c|c|}
\hline Parameter & $p r$ ('mid-c' & $p f($ 'premium & 'fuel-oil' & ') $p f($ ('fuel-gas') & $\overline{p d \text { ('butane }}$ & $p f($ 'distillate') & ('regular & $\begin{array}{l}\text { ') } \\
\operatorname{pr}(\text { ('w-tex') }\end{array}$ \\
\hline rarameter & & P2 & P3 & P4 & P5 & P6 & P7 & \\
\hline$\mu$ & 51.8 & 22.7 & 8.3 & 1.7 & 0.11 & $9.9 \times 10^{-4}$ & 0 & 0 \\
\hline Rank & 1 & 2 & 3 & 4 & 5 & 6 & 7 & 8 \\
\hline
\end{tabular}

In the first step of the sequential reduction, the top four parameters (i.e., $\operatorname{pr}$ ('mid-c'), $p f$ ('premium'), $p f$ ('fuel-oil') and $p f$ ('fuel-gas')) are considered, and the rest 4 parameters are fixed at their nominal values. Hence, a total number of $5^{4}=625$ scenarios are generated based on this setting. The optimal reduction model is applied to these 625 scenarios and the objective is to select 125 scenarios from them. The results of this step are shown in Figure 4. In this figure, the probability mass distribution and the cumulative probability distribution are plotted for both the original 625 scenarios and the selected 125 scenarios. 

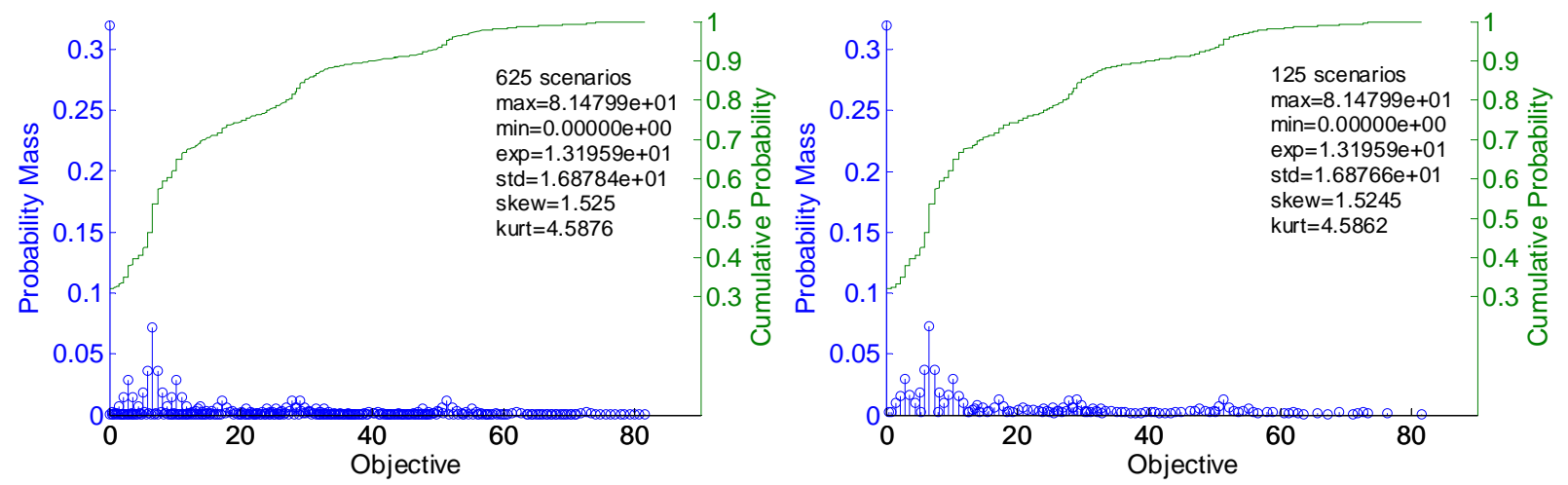

Figure 4. Results of the first step.

Key statistic information including maximum, minimum, expected value, standard deviation, skewness and kurtosis are also presented in Figure 4, which shows that the selected scenarios represent the original scenarios very well. In the next step, the uncertainty in the fifth parameter $p d$ ('butane') is considered and the remaining 3 parameters are fixed at their nominal values. Along with the selected 125 scenarios from the first step, a new set of $125 \times 5=625$ scenarios are generated in this step. Applying a scenario reduction on the new set of scenarios leads to 125 scenarios as shown in Figure 5.
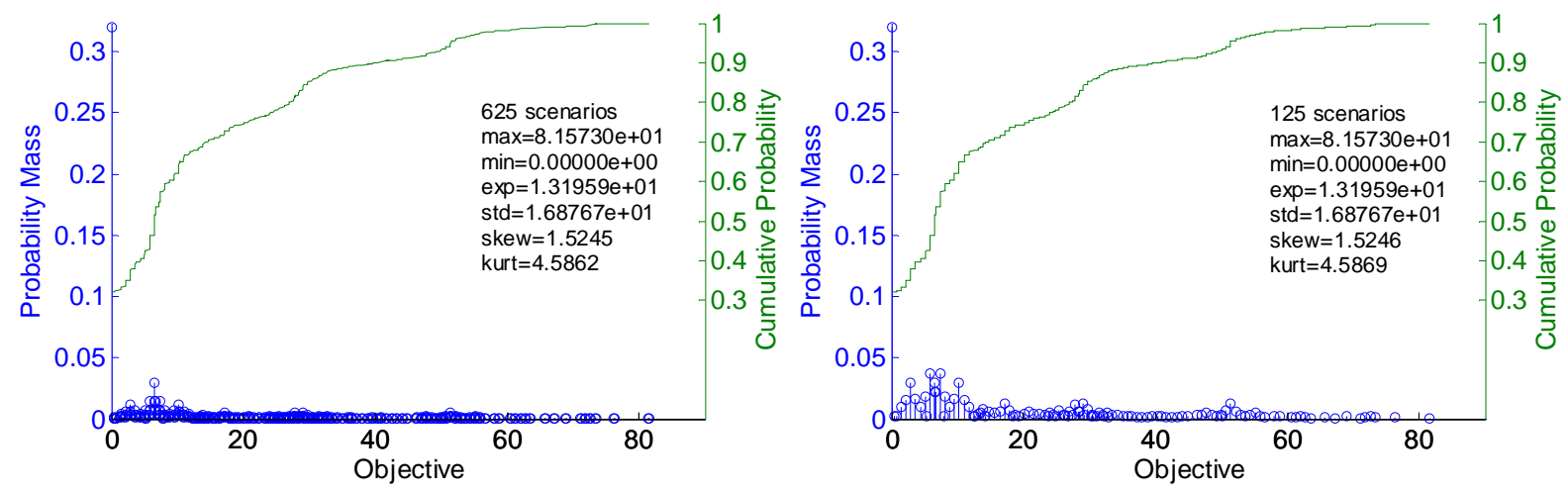

Figure 5. Results of the second step

We repeat the above procedure, until reaching the last parameter $\operatorname{pr}($ 'w-tex'). The results of the final step are shown in Figure 6. 

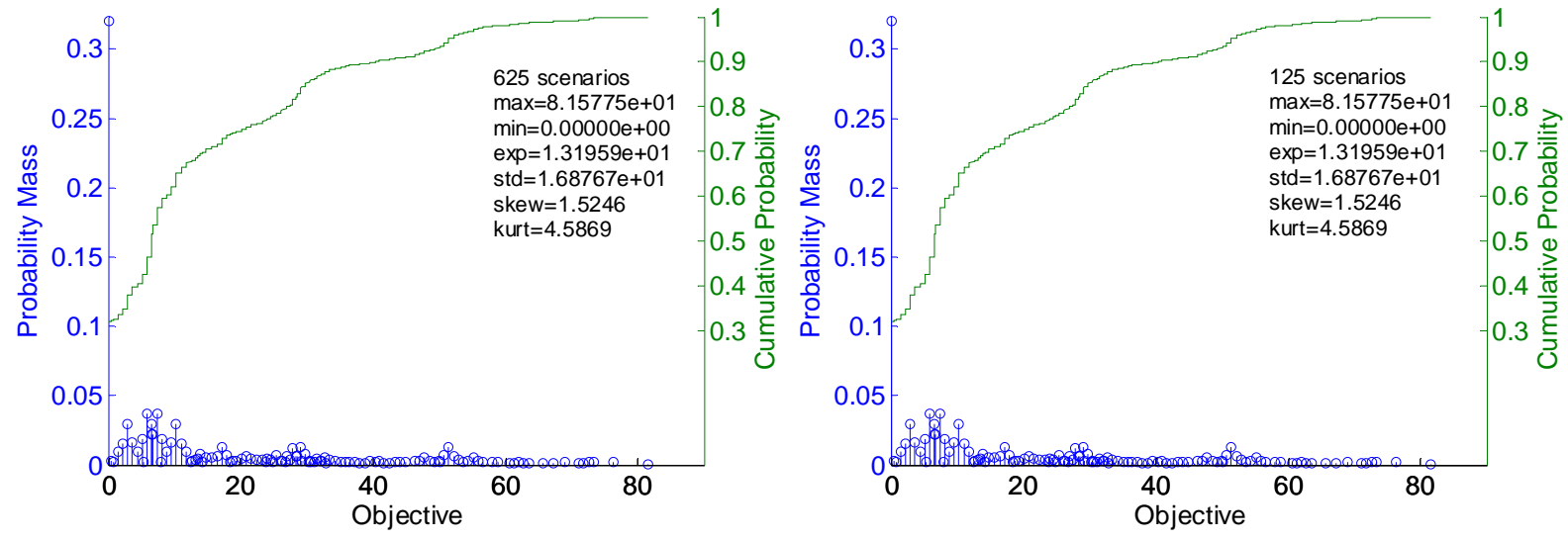

Figure 6. Results of the final step

Finally, the above sequential reduction procedure can be represented by the following Figure 7.

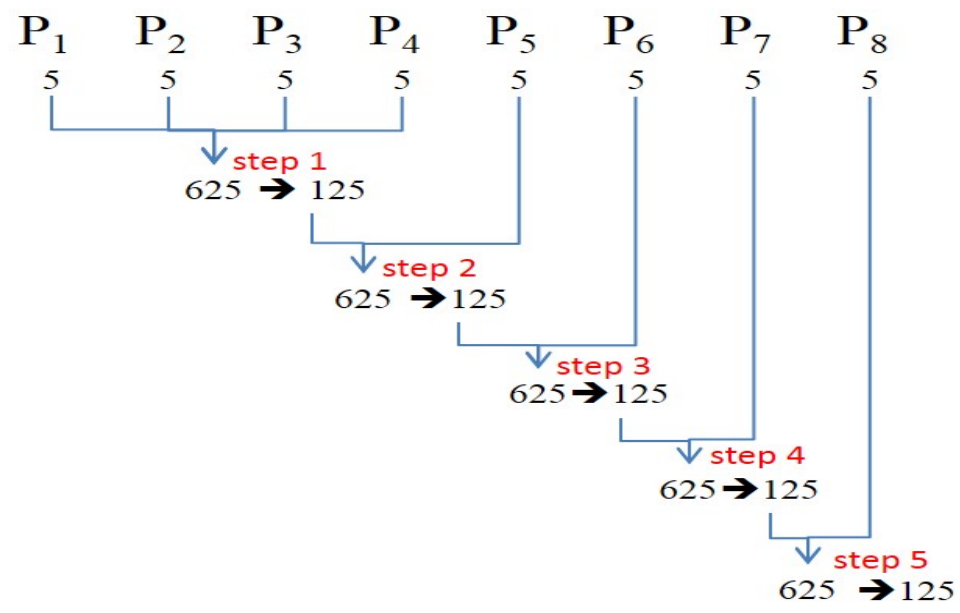

Figure 7. Illustration of the sequential reduction method

To quantify the quality of the scenario reduction, we solve the best performance optimization problem, the robust optimization problem, and the scenario optimization problem and get the following results

$$
\begin{array}{lll}
\hat{f}_{B P}=81.5775 & f_{B P}^{S}=81.57748 & \text { err } r_{1}=0.00002 \\
f_{R O}=0 & f_{S O}^{S}=0 & \text { err } r_{2}=0
\end{array}
$$


Those results demonstrate that the selected scenarios from the sequential framework cover the bounded uncertainty parameter space well, which demonstrates the effectiveness of the sequential scenario reduction approach.

Remark 1. The number of single stage reduction optimization problems is less than the number of parameters and the computational complexity depends linearly on the number of parameters. Hence, the proposed sequential scenario reduction approach avoids the issue of dimensional explosion.

Remark 2. If the desired number of scenarios is 1000 , then the remaining 7-th and 8-th parameters should be considered simultaneously in the final iteration and $125 \times 5 \times 5=3125$ scenarios are generated. So the final reduction problem will be selecting 1000 scenarios from the 3125 scenarios. In summary, a total of four single scenario reduction problems are solved. For other desired number of scenarios, the similar method can be applied to set up the sequential reduction steps.

\section{Effect of ranking (step 1)}

To study the effect of parameter ranking on the final scenario reduction results, the sequential scenario reduction is performed with a random order of the parameters. Specifically, the following random order of parameters were used: $\mathrm{P} 1=p f$ ('fuel gas'), $\mathrm{P} 2=p f($ 'regular'), $\mathrm{P} 3=p r($ 'w-tex'), $\mathrm{P} 4=p d($ 'butane'), $\mathrm{P} 5=p f($ 'premium'), $\mathrm{P} 6=p f($ 'distillate'), $\mathrm{P} 7=p f($ 'fuel oil'), and $\mathrm{P} 8=\operatorname{pr}($ 'mid-c').

Figure 8 compares the results obtained by using the ranked order (left column) and random order (right column) in sequential scenario reduction. It can be observed that the random order case takes more iterations to converge to a distribution that is close to the final results, whereas for ranked order, the distribution tends to be close to the final distribution even in the early iterations. It can be concluded from this observation that while a number of uncertain parameters exist in an optimization problem, the most important parameters play the major roles in the sequential scenario reduction. In practical applications, if computational resources are limited, it is possible to focus on a subset of most important 
parameters to perform the scenario reduction and the final results are expected to be similar to the scenario reduction results using all the parameters.
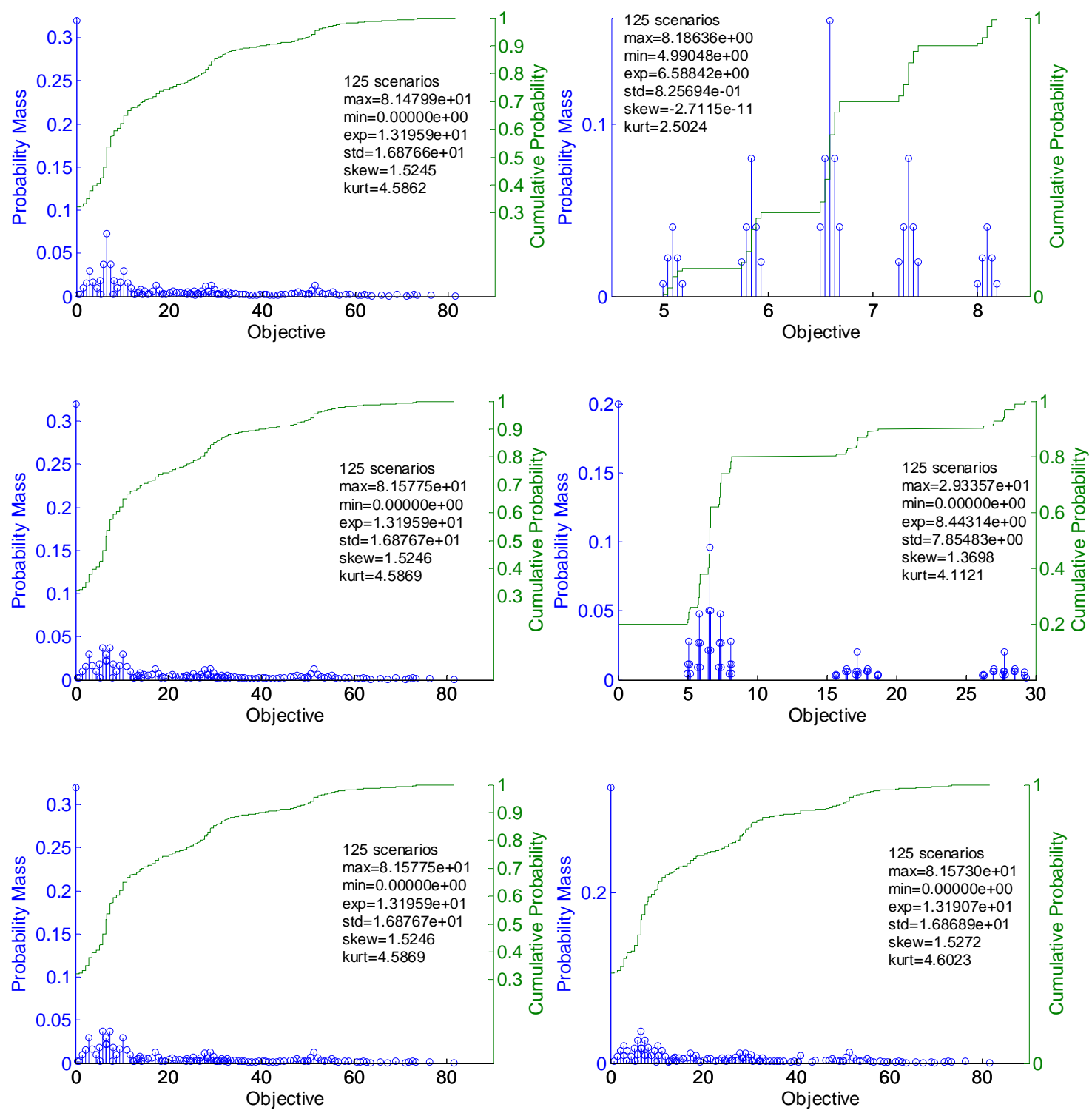

Figure 8. Sequential reduction results. Left: with ranking; Right: with random order Top: first step; Middle, third step; Bottom, fifth step

\section{Effect of number of parameters in the first step}

The sequential procedure can also start from different number of parameters. We run the same problem by starting from 3 parameters in the sequential framework. A comparison of the result for starting from 3 parameters and 4 parameters is shown in Figure 9, it is observed 
that the final results are close in the output performance statistics. The results also show that with more starting number of parameters, the final cumulative distribution of the objective value will be smoother. That is, the final set of scenarios will have a better coverage in the uncertainty space and will lead to a better representation of all the possible number of scenarios.
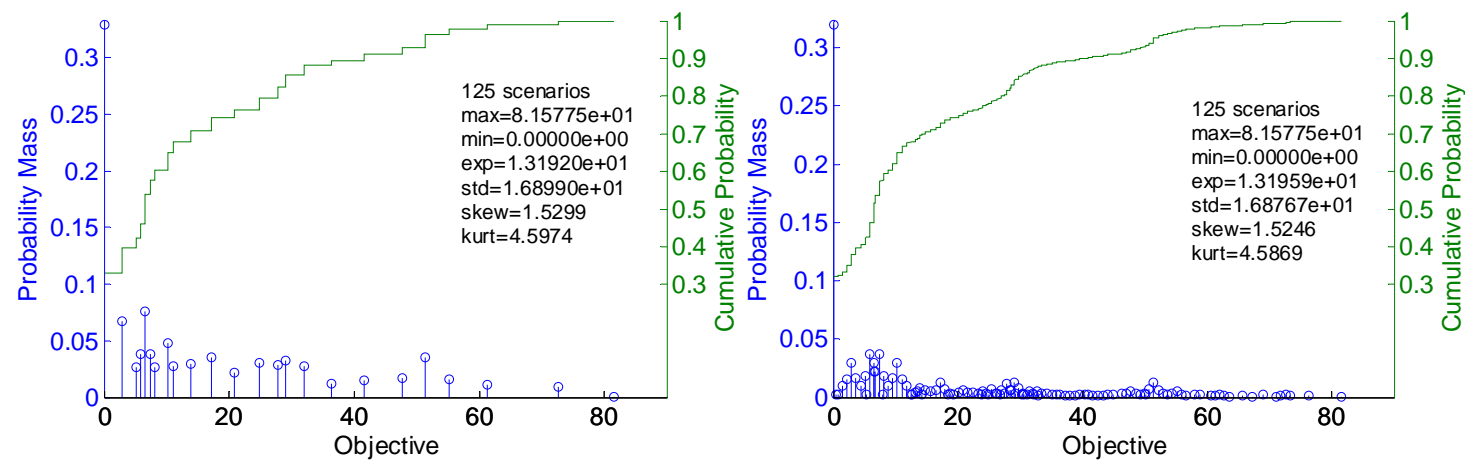

Figure 9. Comparison of different starting number of parameters (Left: Start from 3 parameters, Right: Start from 4 parameters)

Remark 3. Notice that the single scenario reduction is a mixed integer linear optimization problem. To make the sequential reduction efficient, it is preferred that the number of scenarios to be considered in a single reduction step is less than 5000. For example, we should set the initial number of parameters less than 6 , since 6 parameters lead to $5^{6}=15625$ scenarios and that will make the single scenario reduction problem difficult for efficient solution.

\section{Computational studies}

In this section, the proposed sequential reduction method is further studied with a production planning optimization problems. Various numbers of uncertain parameters and discretization levels are investigated to investigate the effectiveness of the proposed method. This example considers the problem of planning the production, storage and marketing of a product for a company. A linear optimization formulation of this problem can be formulated as follows: 


$$
\begin{array}{ll}
\max & \sum_{j} S_{j} z_{j} \\
\text { s.t. } & \sum_{j} C_{j} x_{j}+\sum_{j} V_{j} y_{j} \leq 400,000 \\
& 500+x_{1}-\left(y_{1}+z_{1}\right)=0 \\
& y_{j-1}+x_{j}-\left(y_{j}+z_{j}\right)=0 \quad \forall j=2, \ldots, 6 \\
& y_{6}=500 \\
& x_{j} \leq U_{j} \quad \forall j=1, \ldots, 6 \\
& z_{j} \leq D_{j} \quad \forall j=1, \ldots, 6 \\
& x_{j}, y_{j}, z_{j} \geq 0 \quad \forall j=1, \ldots, 6
\end{array}
$$

In the above model, the decision variables $x_{j}$ represent the production amount during the period $j, y_{j}$ represent the amount of product left in storage (tons) at the end of period $j$ and

\begin{tabular}{|c|c|c|c|c|c|}
\hline$\underset{j}{\text { Period }}$ & $\begin{array}{c}\text { Selling Price } \\
\text { (\$/Ton) } \\
S_{j}\end{array}$ & $\begin{array}{c}\text { Production } \\
\text { Cost (\$/Ton) } \\
C_{j}\end{array}$ & $\begin{array}{c}\text { Storage Cost } \\
\text { (\$/Ton) } \\
V_{j}\end{array}$ & $\begin{array}{c}\text { Production } \\
\text { Capacity (Tons) } \\
U_{j}\end{array}$ & $\begin{array}{c}\text { Demand } \\
\text { (Tons) } \\
D_{j}\end{array}$ \\
\hline 1 & 180 & 20 & 2 & 1500 & 1100 \\
\hline 2 & 180 & 25 & 2 & 2000 & 1500 \\
\hline 3 & 250 & 30 & 2 & 2200 & 1800 \\
\hline 4 & 270 & 40 & 2 & 3000 & 1600 \\
\hline 5 & 300 & 50 & 2 & 2700 & 2300 \\
\hline 6 & 320 & 60 & 2 & 2500 & 2500 \\
\hline
\end{tabular}
$z_{j}$ represent the amount of product sold to market during period $j$. The objective function (15a) maximizes the total sales. The first constraint (15b) ensures that the total cost does not exceed a given budget. Constraints (15c) and (15d) represent the inventory material balances. Constraint (15e) requires that the final inventory meets the desired level (i.e., 500 tons). Constraints (15f) and (15g) represent the production capacity limitations and demand upper bounds, respectively. Nominal data for the above problem are shown in Table 4.

Table 4. Problem data for the production planning problem

\section{Case Study 1. 12 uncertain parameters}

In this case, it is assumed that the 12 parameters $S_{1}$ to $S_{6}$ and $D_{1}$ to $D_{6}$ are subject to independent uncertainty. As shown in Table 5, each uncertain parameter takes 3 possible levels (i.e., low, nominal and high) and each level has a corresponding probability value. 
With a factorial combination, the total number of scenarios is $3^{12}=531441$ and each scenario has a known probability level which can be calculated from the given information. In this study, 243 scenarios will be selected from the 531441 scenarios and new probabilities will be assigned to the selected scenarios.

Table 5. Uncertainty description

\begin{tabular}{|ccc|}
\hline Parameter & Uncertainty level & Probability \\
\hline$S_{1}$ & {$\left[\begin{array}{llll}80 & 180 & 280\end{array}\right]$} & {$\left[\begin{array}{lll}0.3 & 0.4 & 0.3\end{array}\right]$} \\
$S_{2}$ & {$\left[\begin{array}{lll}80 & 180 & 280\end{array}\right]$} & {$\left[\begin{array}{lll}0.3 & 0.4 & 0.3\end{array}\right]$} \\
$S_{3}$ & {$\left[\begin{array}{llll}150 & 250 & 350\end{array}\right]$} & {$\left[\begin{array}{lll}0.3 & 0.4 & 0.3\end{array}\right]$} \\
$S_{4}$ & {$\left[\begin{array}{llll}170 & 270 & 370\end{array}\right]$} & {$\left[\begin{array}{lll}0.3 & 0.4 & 0.3\end{array}\right]$} \\
$S_{5}$ & {$\left[\begin{array}{llll}200 & 300 & 400\end{array}\right]$} & {$\left[\begin{array}{lll}0.3 & 0.4 & 0.3\end{array}\right]$} \\
$S_{6}$ & {$\left[\begin{array}{llll}220 & 320 & 420\end{array}\right]$} & {$\left[\begin{array}{lll}0.3 & 0.4 & 0.3\end{array}\right]$} \\
\hline$D_{1}$ & {$\left[\begin{array}{llll}1000 & 1100 & 1200\end{array}\right]$} & {$\left[\begin{array}{lll}0.3 & 0.4 & 0.3\end{array}\right]$} \\
$D_{2}$ & {$\left[\begin{array}{llll}1000 & 1500 & 2000\end{array}\right]$} & {$\left[\begin{array}{lll}0.3 & 0.4 & 0.3\end{array}\right]$} \\
$D_{3}$ & {$\left[\begin{array}{llll}800 & 1800 & 2800\end{array}\right]$} & {$\left[\begin{array}{lll}0.3 & 0.4 & 0.3\end{array}\right]$} \\
$D_{4}$ & {$\left[\begin{array}{llll}1000 & 1600 & 2200\end{array}\right]$} & {$\left[\begin{array}{lll}0.3 & 0.4 & 0.3\end{array}\right]$} \\
$D_{5}$ & {$\left[\begin{array}{llll}1500 & 2300 & 3000\end{array}\right]$} & {$\left[\begin{array}{llll}0.3 & 0.4 & 0.3\end{array}\right]$} \\
$D_{6}$ & {$\left[\begin{array}{llll}2000 & 2500 & 3000\end{array}\right]$} & {$\left[\begin{array}{lll}0.3 & 0.4 & 0.3\end{array}\right]$} \\
\hline
\end{tabular}

To apply the sequential scenario reduction method, the 12 parameters are first ranked using the Morris method and the result is shown in Table 6. The 12 parameters are labeled as P1 to P12 based on the descending order of importance.

Table 6. Parameter ranking results

\begin{tabular}{|c|cccccccccccc|}
\hline Parameter & $S_{1}$ & $S_{2}$ & $S_{3}$ & $S_{4}$ & $S_{5}$ & $S_{6}$ & $D_{1}$ & $D_{2}$ & $D_{3}$ & $D_{4}$ & $D_{5}$ & $D_{6}$ \\
\hline$\mu\left(\times 10^{5}\right)$ & 1.97 & 2.90 & 3.64 & 3.21 & 4.42 & 5.02 & 0.24 & 1.06 & 3.72 & 2.46 & 3.31 & 2.53 \\
\hline Rank & 10 & 7 & 4 & 6 & 2 & 1 & 12 & 11 & 3 & 9 & 5 & 8 \\
\hline
\end{tabular}

Based on the ranking results, the sequential scenario reduction is applied. Initially, 6 most important parameters (i.e., $S_{6}, S_{5}, D_{3}, S_{3}, D_{5}$ and $S_{4}$ ) are considered and the rest parameters are fixed at nominal values. In this step, the number of generated scenarios is $3^{6}=729$ and 243 scenarios were selected using the optimal scenario reduction method. Similarly, each consecutive step selects 243 scenarios until we reach the 12-th parameter $D_{1}$. 

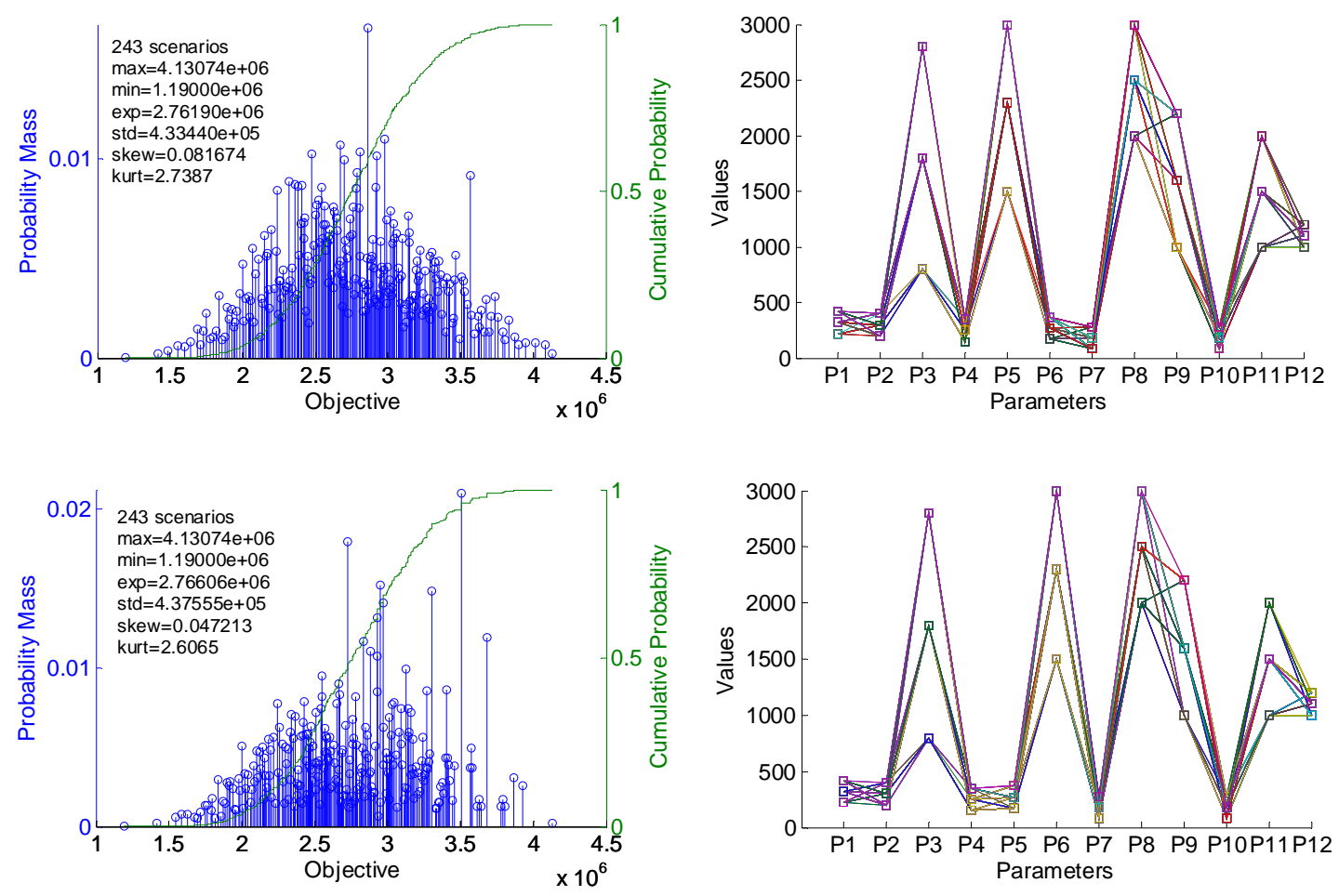

Figure 10. Scenario reduction results

Top: starting from 6 parameters; Bottom: starting from 5 parameters

Starting from 5 parameters, the final results from the sequential reduction procedure is similar (detailed results is given in the supporting information) as shown in Figure 10 (the selected scenarios are also visualized by connecting the different levels of each parameters). To quantify the quality of the scenario reduction, we solve the best performance optimization problem, the robust optimization problem, and the scenario optimization problem and get the following results

$$
\begin{array}{lll}
\hat{f}_{B P}=4.130739 \times 10^{6} & f_{B P}^{S}=4.130739 \times 10^{6} & \text { err } 1=0 \\
f_{R O}=1.19 \times 10^{6} & f_{S O}^{S}=1.19 \times 10^{6} & \text { err } \\
2 & =0
\end{array}
$$

The above results show that the sequential scenario reduction approach leads to the same performance as using the whole uncertainty space, either in best performance optimization or in robust/scenario optimization framework. In this sense, it is a good reduction from the original superset of scenarios.

A sequential reduction with random parameter order was also studied for comparison. In Figure 11, we plotted the maximum objective value among the selected scenarios from each 
iteration step for both ranked case and random order case. The profile shows that the maximum objective value expands as the iteration goes on. After the fourth step, this value tends to be constant for the ranking case, which means that the parameters considered after that step do not affect the maximum objective value of the optimization problem significantly, which is consistent with the global sensitivity analysis based ranking results. In contrast, the results of random ordered parameters will only reach the same level of objective after all the parameters have been covered in the sequential reduction procedure.

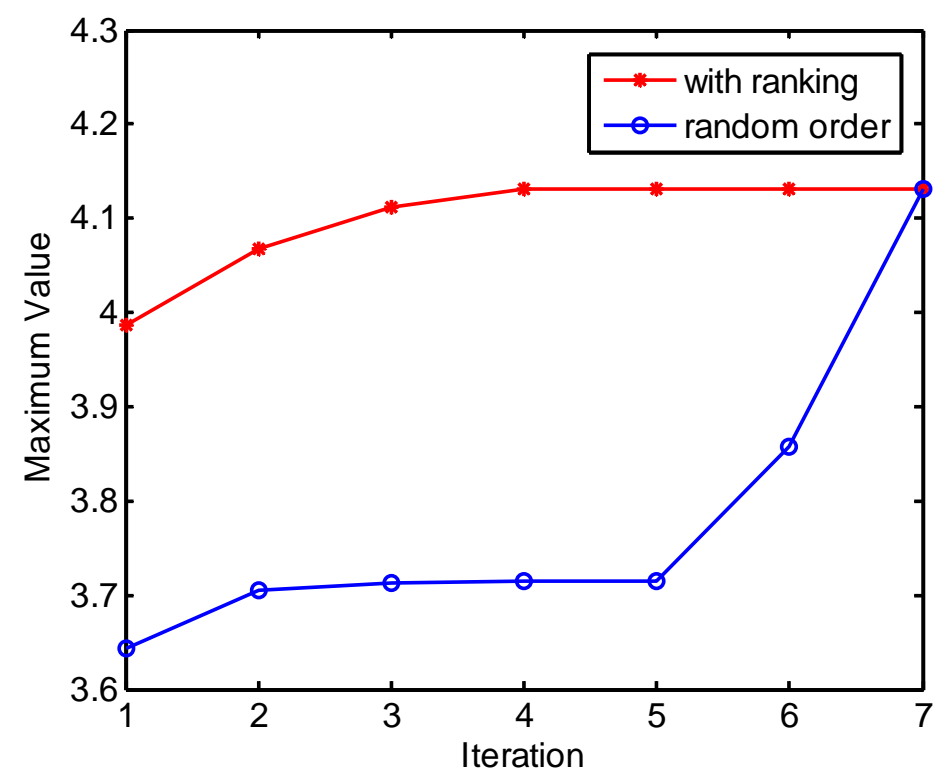

Figure 11. Comparison of the maximum objective value in sequential reduction

\section{Case Study 2. 30 uncertain parameters}

In this test, we consider the case that all the 30 parameters listed in Table 4 are subject to uncertainty and each parameter takes 5 possible levels: nominal value $\times\left[\begin{array}{lllll}0.4 & 0.7 & 1.0 & 1.3 & 1.6\end{array}\right]$. Equal probability $\left[\begin{array}{lllll}0.2 & 0.2 & 0.2 & 0.2 & 0.2\end{array}\right]$ is assumed for the 5 levels for all the parameters. Thus, the total number of scenarios will be $5^{30} \approx 9.31 \times 10^{20}$. Global sensitivity results generated from the Morris method are listed in Table 7. The proposed sequential scenario reduction approach is then performed based on the ranking. 
Table 7. Ranking results for the 30 parameters

\begin{tabular}{|ccc|ccc|ccc|}
\hline parameter & $\mu\left(\times 10^{5}\right)$ & rank & parameter & $\mu\left(\times 10^{5}\right)$ & rank & parameter & $\mu\left(\times 10^{5}\right)$ & rank \\
\hline$S_{1}$ & 2.11 & 11 & $V_{1}$ & 0.02 & 29 & $D_{1}$ & 1.60 & 12 \\
$S_{2}$ & 2.94 & 9 & $V_{2}$ & 0.042 & 28 & $D_{2}$ & 2.17 & 10 \\
$S_{3}$ & 5.59 & 5 & $V_{3}$ & 0.05 & 26 & $D_{3}$ & 4.09 & 8 \\
$S_{4}$ & 5.27 & 6 & $V_{4}$ & 0.06 & 25 & $D_{4}$ & 4.23 & 7 \\
$S_{5}$ & 6.97 & 4 & $V_{5}$ & 0.044 & 27 & $D_{5}$ & 7.23 & 3 \\
$S_{6}$ & 9.19 & 1 & $V_{6}$ & 0.008 & 30 & $D_{6}$ & 9.17 & 2 \\
$C_{1}$ & 0.27 & 24 & $U_{1}$ & 1.04 & 14 & & & \\
$C_{2}$ & 0.39 & 22 & $U_{2}$ & 0.53 & 18 & & & \\
$C_{3}$ & 0.63 & 17 & $U_{3}$ & 0.49 & 20 & & & \\
$C_{4}$ & 1.26 & 13 & $U_{4}$ & 0.51 & 19 & & & \\
$C_{5}$ & 0.73 & 15 & $U_{5}$ & 0.33 & 23 & & & \\
$C_{6}$ & 0.72 & 16 & $U_{6}$ & 0.41 & 21 & & & \\
\hline
\end{tabular}
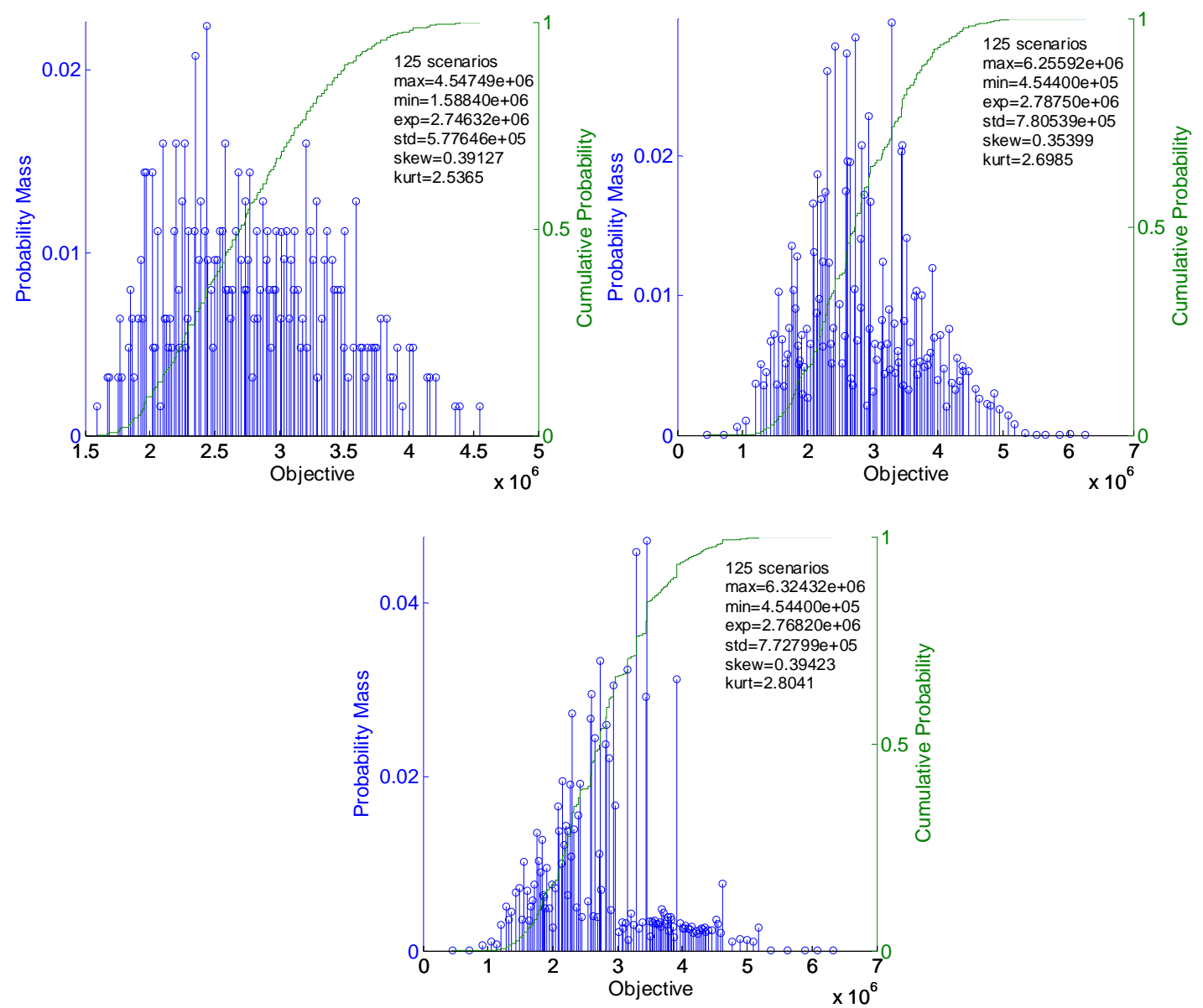

Figure 12. Partial results of the sequential reduction

Top Left: Frist step; Top Right: $13^{\text {rd }}$ step; Bottom: final step 
In this case study, we make the following settings for the sequential reduction procedure. Based on the ranking, the top 4 parameters are initially selected to generate scenarios while the rest 26 parameters are fixed at their nominal value. From the resulting $5^{4}=625$ scenarios, we select 125 of them and take the $5^{\text {th }}$ ranked parameters to generate again 625 scenarios for reduction. This procedure is repeated until we reach the $29^{\text {th }}$ parameter. In the last step, 125 scenarios were selected. Part of the intermediate solution for this case study is illustrated in Figure 12. The results again illustrate the good quality of scenario reduction in each individual step since the performance of the optimization problem is consistently recovered. It is worth mentioning that if the desired number of scenarios is not 125 , it can still be easily handled in the sequential step. For example, if 1000 scenario is preferred, then at the last step, the $29^{\text {th }}$ and the $30^{\text {th }}$ parameter are considered simultaneously so that 3125 scenarios are generated, and 1000 scenarios are selected from them as the final solution.

To quantify the quality of the sequential scenario reduction approach, we solve the best performance optimization problem, the robust optimization problem, and obtain the scenario optimization problem and get the following results:

$$
\begin{array}{lll}
\hat{f}_{B P}=7.2704 \times 10^{6} & f_{B P}^{S}=6.54032 \times 10^{6} & \text { err } \\
f_{R O}=4.544 \times 10^{5} & f_{S O}^{S}=4.544 \times 10^{5} & e r r_{2}=0
\end{array}
$$

For the best performance optimization, the relative gap between the selected scenarios and the best possible value is around $10.04 \%$. That also means that the gap between the best objective from the selected 125 scenarios and the best objective from $5^{30}$ scenarios is less than $10.04 \%$, which shows the good quality of the sequential reduction results.

\section{Conclusion}

In this paper, we extended the optimal single stage scenario reduction method proposed in (Li and Floudas, 2014) to address general optimization problems with a large number of uncertainty scenarios generated from the factorial combination. While the capability of the single scenario reduction is limited by the size of the scenarios, the proposed sequential 
reduction method can be applied to address the huge number of scenarios generated from a large number of uncertain parameters. The proposed method is illustrated through a refinery production optimization and investigated with a production planning problem. We also proposed novel criteria to quantify the quality of scenario reduction when the number of original scenarios is too huge to be evaluated for quality checking. Based on the authors' knowledge, there does not exist a method and readily available tool for scenario reduction from a huge number of scenarios. The proposed method utilizes a rigorous single stage optimal scenario reduction algorithm and a novel sequential framework to address this problem. Although the method is designed for optimization problems, it has many potential applications in reducing uncertainty scenarios for other applications, which will be investigated in future work.

\section{Acknowledgements}

The authors gratefully acknowledge financial support from the National Institute of Health (5R01LM009338) and National Science Foundation (0941143).

\section{References}

[1] Z. Li, C.A. Floudas, Optimal scenario reduction algorithm considering distance of uncertainty distribution and output performance: I. Single reduction via mixed integer linear optimization, Computers and Chemical Engineering, 2014;70:50-66.

[2] G.C. Pflug, Scenario tree generation for multiperiod financial optimization by optimal discretization, Mathematical Programming, 2001;89:251-271.

[3] R. Hochreiter, G.C. Pflug, Financial scenario generation for stochastic multi-stage decision processes as facility location problems, Annals of Operations Research, 2007;152:257-272.

[4] R. Mirkov, G.C. Pflug, Tree approximations of dynamic stochastic programs, SIAM Journal on Optimization, 2007;18:1082-1105.

[5] H. Heitsch, W. Romisch, Scenario tree modeling for multistage stochastic programs, Mathematical Programming, 2009;118:371-406. 
[6] T. Pennanen, Epi-convergent discretizations of multistage stochastic programs via integration quadratures, Mathematical Programming, 2009;116:461-479.

[7] G.C. Pflug, Version-Independence and Nested Distributions in Multistage Stochastic Optimization, SIAM Journal on Optimization, 2009;20:406-1420.

[8] J. Dupacova, N. Growe-Kuska, W. Romisch, Scenario reduction in stochastic programming - An approach using probability metrics, Mathematical Programming, 2003;95:493-511.

[9] H. Heitsch, W. Romisch, Scenario reduction algorithms in stochastic programming, Computational Optimization and Applications, 2003;24:187-206.

[10] H. Heitsch, W. Romisch, A note on scenario reduction for two-stage stochastic programs, Operations Research Letters, 2007;35:731-738.

[11] R. Henrion, C. Kuchler, W. Romisch, Discrepancy distances and scenario reduction in two-stage stochastic mixed-integer programming, J Ind Manag Optim, 2008;4:363-384.

[12] R. Henrion, C. Kuchler, W. Romisch, Scenario reduction in stochastic programming with respect to discrepancy distances, Computational Optimization and Applications, 2009;43:67-93.

[13] H. Heitsch, W. Romisch, C. Strugarek, Stability of multistage stochastic programs, SIAM Journal on Optimization, 2006;17:511-525.

[14] J. Sumaili, H. Keko, V. Miranda, Z. Zhou, A. Botterud, J. Wang, Finding representative wind power scenarios and their probabilities for stochastic models, in: 16th International Conference on Intelligent System Application to Power Systems (ISAP), 2011.

[15] M. Xue, S. Roy, S. Zobell, Y. Wan, C. Taylor, C. Wanke, A stochastic spatiotemporal weather-impact simulator: Representative scenario selection, in: AIAA Aviation Technology, Integration, and Operations (ATIO) Conference, 2011.

[16] J.P.d. Costa, G.C.d. Oliveira, L.F.L. Legey, Reduced scenario tree generation for midterm hydrothermal operation planning, in: 9th International Conference on Probabilistic Methods Applied to Power Systems, 2006.

[17] R. Karuppiah, M. Martin, I.E. Grossmann, A simple heuristic for reducing the number of scenarios in two-stage stochastic programming, Computers \& Chemical Engineering, 2010;34:1246-1255. 
[18] W.L. de Oliveira, C. Sagastizabal, D.D.J. Penna, M.E.P. Maceira, J.M. Damazio, Optimal scenario tree reduction for stochastic streamflows in power generation planning problems, Optim Method Softw, 2010;25:917-936.

[19] M.D. Morris, Factorial Sampling Plans for Preliminary Computational Experiments, Technometrics, 1991;33:161-174.

[20] I.M. Sobol. Sensitivity estimates for nonlinear mathematical models. Mathematical Modelling and Computational Experiments, 1993;14:407-414

[21] R.I. Cukier, C.M. Fortuin, K.E. Shuler, A.G. Petschek, J.H. Schaibly, Study of the sensitivity of coupled reaction systems to uncertainties in rate coefficients. I Theory, Journal of Chemical Physics, 1973;59:3873-3878.

[22] A. Saltelli, S. Tarantola, K. Chan, A quantitive model-independent method for global sensitivity analysis of model output, Technometrics, 1999;41:39-56.

[23] D. Kendrick, A. Meeraus, J.S. Suh, Oil refinery modeling with the GAMS language, in Tech. Rep., Center for Energy Studies, The University of Texas, 1981.

[24] Calfa, Bruno A., et al. Data-driven multi-stage scenario tree generation via statistical property and distribution matching. Computers and Chemical Engineering, 2014;68:723 
Appendix. Nomenclature for the refinery optimization example

\section{Index/Set}

$c \in C \quad$ materials

$c f \in C F \quad$ final product

$c r \in C R \quad$ crude oil

$c i \in C I \quad$ intermediates

$c d \in C D \quad$ domestic products

$p \in P \quad$ processes

$m \in M \quad$ process units

$q \in Q \quad$ quality attributes

\section{Parameter}

$a_{c r, c, p}$

$b_{m, p}$

$k_{m}$

$p d_{c d}$

$p r_{c r}$

$p f_{c f}$

$u r_{c r}$

$o p_{p}$

$q s_{c f, q}^{l b}$

$q s_{c f, q}^{u b}$

$a t_{c i, q}$

$a t c_{c r, c i, q}$

\section{Variables}

$z_{c r, p}$

$x_{c f}$

$u_{c r}$

$u i_{c r, c i}$

$w_{c r, c i, c f}$ input-output coefficients

capacity utilization

initial capacity

prices of domestic products $(\$ / b)$

prices of crude oils $(\$ / b)$

prices of final products $(\$ / b)$

upper bound on crude oil (1000bpd)

operating cost $(\$ / b)$

product quality specifications lower bound

product quality specifications upper bound

attributes for blending

attributes for crude blending

process level

final sales

purchase of crude oil

purchase of intermediate materials

blending process level 